EPJ manuscript No.

(will be inserted by the editor)

\title{
Dynamics of cluster deposition on Ar surface
}

\author{
P. M. Dinh ${ }^{1}$, F. Fehrer ${ }^{2}$, P.-G. Reinhard ${ }^{2}$, and E. Suraud ${ }^{1}$ \\ 1 Laboratoire de Physique Théorique, UMR 5152, Université P. Sabatier, F-31062 Toulouse cedex, France \\ ${ }^{2}$ Institut für Theoretische Physik, Universität Erlangen, Staudtstrasse 7, D-91058 Erlangen, Germany
}

November 10, 2018/ Received: date / Revised version: date

\begin{abstract}
Using a combined quantum mechanical/classical method, we study the dynamics of deposition of small $\mathrm{Na}$ clusters on $\operatorname{Ar}(001)$ surface. We work out basic mechanisms by systematic variation of substrate activity, impact energy, cluster orientations, cluster sizes, and charges. The soft Ar material is found to serve as an extremely efficient shock absorber which provides cluster capture in a broad range of impact energies. Reflection is only observed in combination with destruction of the substrate. The kinetic energy of the impinging cluster is rapidly transfered at first impact. The distribution of the collision energy over the substrate proceeds very fast with velocity of sound. The full thermalization of ionic and atomic energies goes at a much slower pace with times of several ps. Charged clusters are found to have a much stronger interface interaction and thus get in significantly closer contact with the surface.
\end{abstract}

PACS. 36.40.Gk, 36.40.Mr,36.40.Sx, 36.40.Vz, 61.46.Bc Atomic and molecular clusters

\section{Introduction}

Clusters on surfaces are an appealing area of research ' which have motivated numerous studies [1]. The litera' ture on the topic is flourishing and many conferences have been (fully or partly) devoted to these researches, see for ' example the series of recent ISSPIC conferences [2,3,4, 5. It is now possible to make a direct deposition of size selected clusters on a substrate 6, 7. This opens up new possibilities for the synthesis of nano-structured surfaces. But the deposition process is not necessarily simple and it may lead to a significant modification of the cluster, both in terms of its electronic structure and of its ionic geometry. There is a subtle interplay of the interface energy, electronic band structure of the substrate, and surface corrugation. These various aspects have already been investigated in great detail, especially from the structural point of view, both experimentally [8, 9, 10 and theoretically [11, 12,13, 14, 15, 16, 17, 18]. The situation becomes even more involved when one considers the deposition dynamics itself. But that makes the case also more interesting. And in a such involved situation, one should start to disentangle the various influences by studying simple systems, i.e. simple geometries and material combinations. In this contribution, we are presenting first results for the deposition dynamics of small Na clusters on $\operatorname{Ar}(001)$ surface. To that end, we employ a hierarchical model which treats the different subsystems at different levels of refinement, depending on their relevance for the whole process.

While the presence of a substrate makes the experimental handling of clusters easier, it strongly complicates the theoretical description because of the huge num- ber of degrees of freedom of the surface. Most theoretical approaches thus remain limited to molecular dynamics (MD). The MD provides the cheapest way to access, at least in a gross way, the dynamics of the substrate. However, its applicability remains restricted to a narrow range of physical situations, because a proper description of electronic degrees of freedom is missing. It is nevertheless crucial to try to account for the surface electronic degrees of freedom, in particular if non-adiabatic processes become involved.

A first simplification is to use relatively simple cluster/substrate combinations as, e.g., simple metal cluster on an insulator surface. Because of its inert nature the insulator surface can be included at a lower level of description. This was, e.g., explored for the case of Na clusters on $\mathrm{NaCl}$ in [18, 19] where the cluster electrons were described fully quantum-mechanically while the substrate is frozen and just serves to deliver an effective interface potential (itself tuned to $a b$-initio calculations [14). However rare-gas atoms show sizeable polarization response. These effects need to be included. A somewhat better description of surface degrees of freedom can be achieved by allowing for a minimum of dynamical response of the substrate, as recently proposed for the description of $\mathrm{Na}$ clusters embedded in rare gases $20,21,22$. The electronic degrees of freedom of the cluster were treated microscopically by time-dependent density-functional theory while $\mathrm{Na}$ ions and substrate atoms were handled with classical MD, taking carefully into account the dynamical polarization of the atoms. This model belongs to the family of coupled Quantum-Mechanical with Molecular-Mechanical methods (QM/MM) which are often used in other fields 
P. M. Dinh et al: Dynamics of cluster deposition on Ar surface

as, e.g., bio-chemistry 23, 24, 25] or surface physics 26, 27.

In this paper we apply this hierarchical approach to study the deposition of small Na clusters on an Ar surface. The paper is organized as follows: Section 2 provides a brief presentation of the hierarchical approach and the construction of a surface. In section 3, we discuss the effects of the dynamical treatment of the Ar atoms. In section 4. we discuss variation of collisional energy in terms of ionic motion and energetic observables. In section 5 we study the influence of the initial collisional geometry. And in section 6. we look at variation of cluster size and charge.

\section{Model}

We give here a short summary of the model, for more details see appendix $\mathrm{A}$ and 28 . The Na cluster is described in the TDLDA-MD approach, which was well validated for linear and non-linear dynamics of free metal clusters 29, 30. The Na electrons are treated by means of densityfunctional theory at the level of the time-dependent localdensity approximation (TDLDA), and the $\mathrm{Na}^{+}$ions are propagated by means of molecular dynamics (MD). The electron-ion interaction is described by soft, local pseudopotentials 31. Each Ar atom has two classical degrees of freedom: center-of-mass and electrical dipole moment. The dipoles allow an explicit treatment of the dynamical polarizability of the atoms. The key constituent of the dynamic cluster-atom interaction are polarization potentials 32. A local pseudo-potential is added for the electron-Ar short-range repulsion, modeled following [33, and with a final slight readjustment to the NaAr dimer as benchmark (bond length, binding energy, and optical excitation). The Na-Ar Van-der-Waals interaction is computed via the variance of dipole operators $20,28,33$. The atom-atom interactions are described by a standard Lennard-Jones potential, while the $\mathrm{Ar}-\mathrm{Na}^{+}$subsystem is treated by means of effective potentials from the literature 34. More details on these various components, including the choice of parameters can be found in appendix.

The total energy thus composed is the starting point for variation leading to the time-dependent Kohn-Sham equations for the cluster electrons coupled with Hamiltonian equations of motion for the classical degrees of freedom $\left(\mathrm{Na}^{+}\right.$ions, Ar positions and dipoles). The initial condition is obtained from solving the corresponding stationary equations and finally boosting the $\mathrm{Na}$ cluster to the wanted impact energy.

The numerical solution proceeds with standard methods 30. We use space-grid techniques to solve the (timedependent) Kohn-Sham equations for the cluster electrons. The time propagation is based on a time-splitting method, and the stationary solution is attained by accelerated gradient iterations. The electronic mean-field is treated in axially averaged approximation [35, 36, which was found to provide an acceptable approximation for the present test case of the nearly axial $\mathrm{Na}_{6}$ cluster impinging on a surface. In the following, the symmetry axis will be denoted by the $z$ axis. It also corresponds to the deposition axis. The $\mathrm{Na}^{+}$ions and the Ar atoms are, of course, treated in full three dimensions.

Several observables can be computed. We employ here simple geometric and energetic indicators, the ionic/atomic positions and their kinetic energies. We have checked also electronic observables, as dipole oscillations and ionization. Both play a minor role for the analysis (e.g., electronic emission during deposition is less than $0.02 \%$ ). However, this does not imply that the dynamical path evolves along a simple and geometrically prescribed BornOppenheimer surface. Cluster electrons and ions couple to the many atoms in the surface through short range collision and long-range dynamical polarizability which excites all degrees of freedom in a state far from equilibrium.

Our main test case is $\mathrm{Na}_{6}$ on Ar surface. $\mathrm{Na}_{6}$ consists out of five $\mathrm{Na}$ ions in a ring plus one 6th ion topping the ring. The top ion sits on the symmetry axis. The $\operatorname{Ar}(001)$ surface is modeled as six layers of $8 \times 8$ squares containing together $384 \mathrm{Ar}$ atoms. The squares are copied periodically in both horizontal directions to simulate an infinite surface. To stabilize the underlying (supposedly infinite) crystal structure, the atomic positions in the lower two layers are frozen at the bulk positions. Some comparisons have been performed with the larger substrate configuration $\mathrm{Ar}_{512}$, composed by 6 active layers and 2 frozen ones. We have found good agreement, justifying that 4 active layers are sufficient in the present dynamical regime. Finally in our energetic analysis, the Ar atoms in the vicinity of the impact point will be emphasized. This includes an hemisphere around the impact point $(4 \times 4$ in first layer, $3 \times 3$ in second, and $2 \times 2$ in third). We will see that this impact hemisphere carries almost the whole energy transfer in the deposition process (cf. right panel of Fig. 3).

\section{The effect of the surface}

In a first run, we investigate the influence of the modeling of the Ar substrate. The $\mathrm{Na}_{6}$ center-of-mass starts from $15 a_{0}$ above the surface with an initial velocity in the $z$ direction, corresponding to a kinetic energy $E_{\text {kin }}^{0}$ of 0.06 Ry. Experimentally, this setup should lead to sticking, if one extrapolates available experimental data with proper scaling laws (see the discussion below) [37. In order to analyze the impact of the various ingredients of the model, Fig. 1 compares the deposition dynamics for three different levels of treatment, full model, dynamical dipoles switched off, and frozen atomic positions (while maintaining dynamical dipoles). The cluster is initially in "bottom" configuration where the pentagon is closer to the surface and the top ion faces away. In all three cases, the first $500 \mathrm{fs}$ of the dynamics proceed similar showing the cluster steadily approaching the surface. Then the collision and the subsequent evolution proceed very differently. For the fixed Ar atoms (lower right panel), the surface reacts rigidly and one observes an immediate reflection of $\mathrm{Na}_{6}$ combined with strong internal excitations as oscillations of the top ion through the pentagon. For mobile Ar atoms without dipole dynamics (lower left panel), there is again 

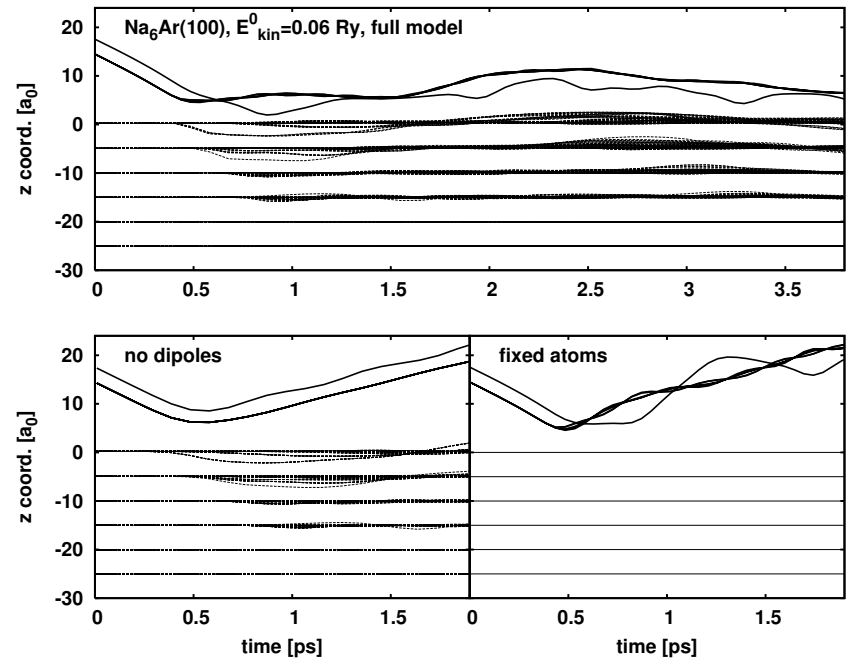

Fig. 1. $z$ coordinates of the $\mathrm{Na}_{6}$ cluster (full curves) deposited on $\mathrm{Ar}_{384}$ (dotted) for an impact energy of $0.06 \mathrm{Ry}$. Three different levels of treatment are shown. Upper panel: full model. Lower left panel: dynamical dipoles of Ar atoms switched off. Lower right panel: totally frozen Ar atoms.

reflection, but now with a visible amount of excitation energy going to the substrate while less is left for internal motion of the cluster. For the case of fully active Ar atoms (upper panel), the substrate absorbs most of the cluster's kinetic energy and the cluster remains tied to the surface, with its distance to the surface performing large oscillations in the attractive potential well created by the Ar dipoles. We thus observe a relatively soft landing of the $\mathrm{Na}_{6}$ in "bottom" configuration. Soft means here that the surface and the cluster are still moderately perturbed. The overall comparison of the three calculations demonstrates the importance of a full dynamical treatment of the Ar surface, going beyond a mere Molecular Dynamics of Ar positions but also accounting for their polarizabilities through time-dependent dipoles.

The perturbation produced by the impact of the cluster on the first layer propagates straightforwardly through the substrate as a sound wave with about the speed of sound inside Ar bulk, namely around 20-30 a $/$ ps. When this sound wave reaches the 5th (and frozen) layer, it is reflected upwards. When coming back to the surface it transfers some residual momentum to the cluster. However that momentum transfer remains moderate and leaves the cluster in a captured state. That back-flow of momentum would not show up in that pronounced manner for an infinitely deep material. We have checked that in this energy range and in the case of the deposit of a single $\mathrm{Na}$ atom, adding 2 more Ar layers. This indeed does not change the scenario qualitatively as the 2 added active Ar shells do absorb a rather limited amount of kinetic energy. It might nevertheless modify in fine the deposition threshold quantitatively. However the model with frozen bottom layers has a realistic touch. It provides a zeroth-order simulation of surfaces from hard materials covered by a thin layer of rare gas 38 . Mind nevertheless that the case may differ at a quantitative level. In the case of Ar coated metals, for example, the underlying metal provides an extra attraction on the deposited cluster. This may thus modify the details of the deposition scenario, especially at the side of energetic considerations and deposition thresholds.

Altogether, Fig. 1]demonstrates the crucial role played by the elasticity of the surface. Ar is extremely soft and serves as a true stopper material for gentle deposition. The proper dynamical treatment of the surface also appears as essential.

\section{Energetic analysis}

In this section, we study the dependence on the initial kinetic energy $E_{\text {kin }}^{0}$ of $\mathrm{Na}_{6}$. This amounts to change its initial velocity along the $z$ direction. Fig. 2 summarizes results for the time evolution viewed through spatial coordinates and kinetic energies. A very broad range of initial kinetic energies $E_{\text {kin }}^{0}$, from $0.6 \mathrm{Ry}$ down to $0.006 \mathrm{Ry}$, is considered. The detailed atomic and ionic $z$ coordinates (left column) show at first glance an overall similarity of all the different cases, except for the highest energy. There is a very fast stopping and quick capture of the Na cluster followed by persistent oscillations of the cluster distance plus some internal oscillations. The cluster momentum transferred at impact propagates as a sound wave through the substrate. The perturbation of the substrate, of course, increases with the initial energy. The case of the highest energy differs. One sees reflection of the cluster, however at the price of severe destruction of the surface. It corroborates the view that $\mathrm{Ar}$ is an extremely efficient stopper material. At this point, a comparison with experiments performed on comparable systems [37] can be made. The measurements of 37] were, in fact, carried out with the much heavier Ag material but once deposition energies are properly scaled, our results are in qualitative agreement. Note that sufficiently high deposition energies were not attained in the experiments of [37] to access the destructive regime. In our simulations, the threshold for destruction of the substrate seems to lie between 0.05 and 0.1 Ry per metal atom. It should finally be noted that for all cases of non-destructive deposition, the course of the process does not depend much on the initial projectile velocity. The same result has been found in studies of deposition on large, but finite, Ar clusters 39.

The kinetic energies are shown in the second column of Fig. 2. The kinetic energy of the $\mathrm{Na}$ cluster increases before contact due to the long-range polarization interaction which is attractive. The additional acceleration depends on the initial velocity. Slower velocities allow for more energy gain, in relative value, since the cluster moves for a longer time in the attractive regime. The kinetic energy is multiplied almost by a factor 8 for the lowest $E_{\text {kin }}^{0}=0.006$ Ry whereas only insignificant acceleration occurs for the fastest collision. At time of impact, there emerges a very fast and almost complete energy transfer from the Na cluster to the substrate. In less than $0.5 \mathrm{ps}$, the Ar carry away almost the whole $\mathrm{Na}$ cluster kinetic energy and only very little residual kinetic energy is left to the $\mathrm{Na}$ cluster. 



Fig. 2. Time evolution of deposition of $\mathrm{Na}_{6}$ on $\mathrm{Ar}_{384}$ for five different initial kinetic energies $E_{\mathrm{kin}}^{0}$ as indicated. Left column: $z$ coordinates of $\mathrm{Na}^{+}$ions (heavy solid line) and of Ar atoms (faint dashed). Right column: kinetic energies for the Na cluster (solid lines) and for the Ar system (dashed).

Longer times are needed for the final relaxation processes. Note also the revival of the cluster kinetic energy a few ps after impact. This is due to the come back of the reflected wave in the substrate, as discussed above.

It is instructive to analyze energy transfers in terms of temperature. To that end, we calculate the intrinsic kinetic energy $E_{\text {kin }}^{\text {int }}$ by subtracting the contribution from the center-of-mass motion which is particularly relevant for the Na cluster. The kinetic temperature is then defined as $T_{\text {kin }}=2 E_{\text {kin }}^{\text {int }} / 3 N$, where $N=6$ for the cluster and 384 for the substrate. The time evolution of the kinetic tempera- ture is plotted in Fig. 3 The first column shows $T_{\text {kin }}$ for the cluster. The initial phase is purely center-of-mass motion without intrinsic excitation. The temperature jumps at the moment of impact due to the large perturbation of all constituents in the impact zone. The jump ends close to the final temperature and there remains a slow and moderate relaxation to thermal equilibrium. The right column of Fig. 3 shows the temperatures for the substrate in two ways, taken over the atoms in the vicinity of the impact point (the "impact hemisphere" as explained at the end of section 2) or over all Ar atoms outside the impact hemi- 

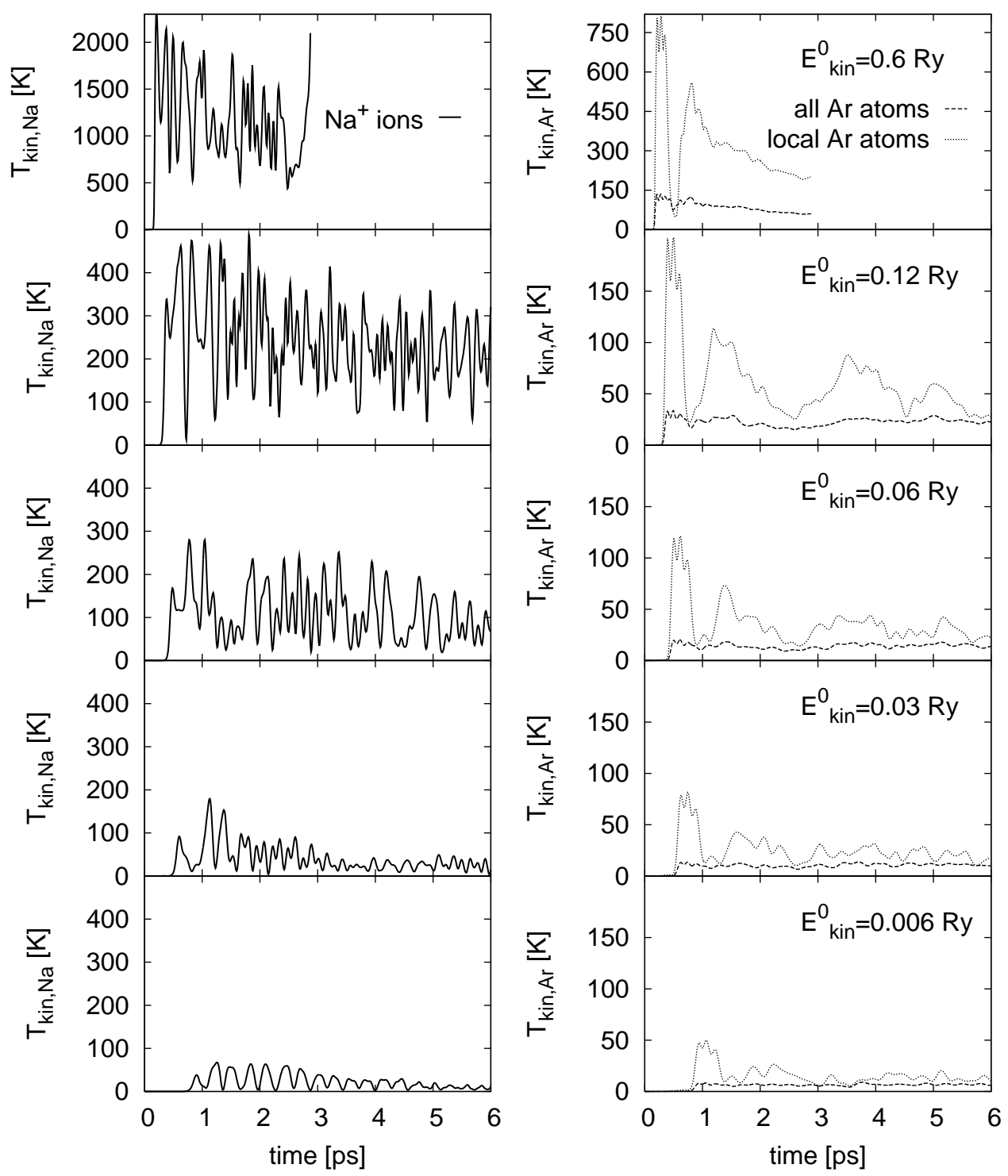

Fig. 3. Time evolution of deposition of $\mathrm{Na}_{6}$ on $\mathrm{Ar}_{384}$ for five different initial kinetic energies $E_{\text {kin }}^{0}$ as indicated (Figure 2 continued). Left column: kinetic temperature of $\mathrm{Na}^{+}$ions. Right column: kinetic temperature of the Ar substrate taken over the impact hemisphere (dashed) denoted as "local" and over all remaining atoms (dotted) denoted "all".

sphere. The differences between these both temperatures are huge. The initial energy transfer goes preferably to the region around the impact point. The kinetic temperature of the impact hemisphere shows recurrent bumps. They are related to slow oscillations within the substrate (see first column of Fig. (2) and associated energy exchange between potential and kinetic energy. The average temperature relaxes slowly to that of the total system. The amplitude of the oscillations and the relaxation time strongly depend on the initial energy, ranging from about $5 \mathrm{ps}$ for the weakly excited cases to outside our simulation time for the heftier processes. Note that the average temperature for the highest energy is above the melting point of about $84 \mathrm{~K}$ [40,41, indicating once more the destruction of the substrate. The electronic excitation during the collision amounts to small dipole oscillation with an amplitude of about $0.05 \mathrm{a}_{0}$ and related energy content of about 6.8 $\mathrm{meV}$.

In the second column of Fig. 2, we had tracked the kinetic energy transfer from the cluster to the surface as a function of time. It is also interesting to study the final repartition of the initial energy. For that purpose, the values of the ionic kinetic, the atomic potential and the atomic kinetic energies after 6 ps are recorded and are normalized to the maximum kinetic energy, $E_{\mathrm{kin}}{ }^{\max }$, reached before impact. The thus obtained energy ratios are plotted as function of $E_{\text {kin }}^{0}$ in Fig. 4 . The figure shows that the energy share left for the Na cluster increases with in- 
creasing deposition energy. Nonetheless, the energy loss at the side of the $\mathrm{Na}$ cluster is dramatic, even for the most violent case. For the Ar substrate, we see an equal share between potential and kinetic energies, except for the highest initial energy. The gain in potential energy is related to the moderate spatial rearrangements in the $\mathrm{Ar}$ substrate. For $E_{\text {kin }}^{0}=0.6 \mathrm{Ry}$, the kinetic energy is $2 / 3$ times larger than the potential one. Indeed the emitted $\mathrm{Ar}$ in this case are blown away with a substantial velocity (see upper left panel of Fig. 2).

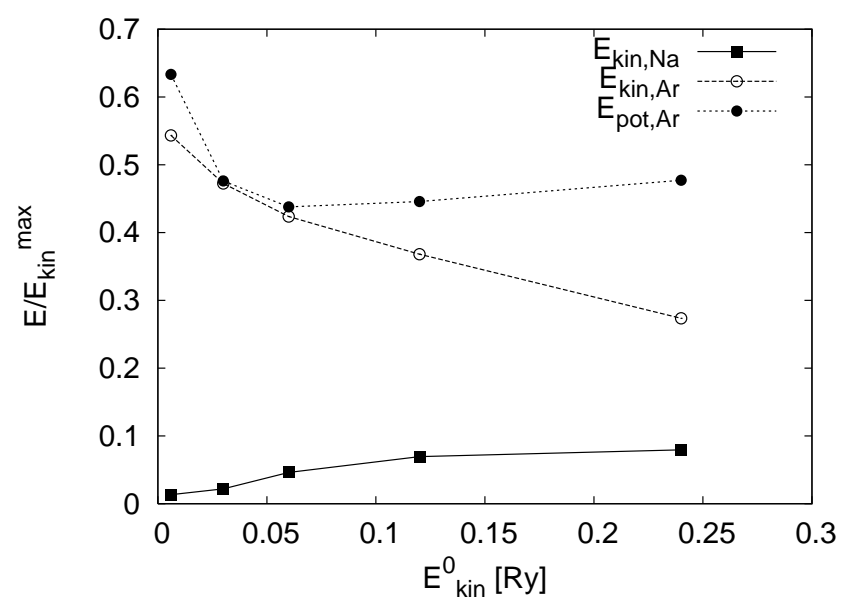

Fig. 4. Partitioning of final energies over cluster kinetic energy, substrate kinetic, and substrate potential energy normalized to initial energy.

\section{Geometrical effects}

There are different possible initial geometries (orientation, position relative to surface) which may produce different reaction pattern (for an example, see the case of $\mathrm{Na}_{6}$ on $\mathrm{NaCl}$ [18, 19]). All results reported above are obtained from the starting configuration displayed on the left col-

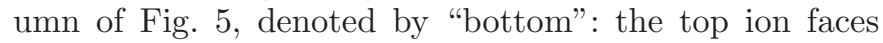
away from the surface and the cluster center axis is placed above an interstitial position of the first Ar layer. Two other configurations have been studied. The "centered" one (middle column of Fig. (5) is similar to the "bottom" configuration but with the axis exactly above an Ar atom of the first layer. The "on top" configuration (right column of Fig. (5) is obtained from the "bottom" configuration by reversing the top ion to face towards the surface such that the top ion hits the Ar surface first. In all cases, the cluster center-of-mass is initially positioned $15 a_{0}$ above the first layer.

The results for the time evolution of the $z$ coordinates and the kinetic energies are shown in Fig. 5 . At first glance, all three results look very similar. Interesting differences become apparent in the details. The average kinetic energy of the cluster is largest for the "bottom" configuration and smallest for "on top". This relates to the amount of
Ar core repulsion presented to the cluster. For "bottom", the axis lies on an interstitial position and the $\mathrm{Na}$ ions stay closer to the sites of the Ar atoms. For "centered", the most repulsive site points in between the $\mathrm{Na}$ ions of the pentagon, the interaction being that way weakened. The situation is somehow similar for "on top" where the now closest top ion dives into the interstitial site with maximum distance to the atoms. This also explains why the top ion does not go through the pentagon in that case. There is also a slight difference in the final distance to the surface. The "on top" configuration seems to stay closer which may be, again, due to a minimization of core repulsion in this configuration.

\section{Size and charge effects}

In the previous sections, we finally observed in most cases the soft landing of the $\mathrm{Na}_{6}$ on the Ar surface. One may suspect that this is favored by the oblate shape of this cluster which provides a large contact area. It is interesting to test the deposition scenario for different cluster geometries. A good alternative is $\mathrm{Na}_{8}$. As is well known the 8 valence electrons of $\mathrm{Na}_{8}$ form a closed shell [42,43] (magic number of electrons), which leads to a close to spherical (up to the impact of ions) electronic shape. The overall shape of $\mathrm{Na}_{8}$ is, in turn, itself close to spherical and is thus significantly different from the especially oblate one of $\mathrm{Na}_{6}$. The left part of Fig. 6 shows the time evolution for $\mathrm{Na}_{8}$ impinging on $\mathrm{Ar}_{384}$ surface. The scenario for $\mathrm{Na}_{8}$ is very similar to what we had observed for $\mathrm{Na}_{6}$, with efficient stopping, sudden energy transfer, and final capture. One even observes less excitation and deformation of the $\mathrm{Na}_{8}$ cluster than in the $\mathrm{Na}_{6}$ cases. This effect is probably due to the fact that $\mathrm{Na}_{8}$ is electronically magic and thus especially stable.

The right part of Fig. [6]shows the result for the charged cluster $\mathrm{Na}_{9}^{+}$. It has also the magic electron number 8 and is near to spherical shape. While the overall bouncing scenario is qualitatively similar (with sound wave bounce), the net result is quantitatively different. Differences show up already in the initial phase. The finite net charge yields stronger attractive polarization interaction with the substrate and thus acquires much more acceleration. The thus higher impact energy and the stronger interaction produce a more violent collision. Most remarkable is the closer attachment to the surface which, again, is due to the much stronger polarization interaction. The bottom of the $\mathrm{Na}_{9}^{+}$ cluster is even merging into the surface layer. This small example indicates that the deposition of charged clusters is a most interesting topic which deserves further studies, also in direct relation with experiments [37,44].

\section{Conclusions}

In this paper, we have investigated the deposition dynamics of small Na clusters on an $\operatorname{Ar}(001)$ surface. To that end, we have employed a hierarchical model treating the $\mathrm{Na}$ 



Fig. 5. Time evolution of $z$ coordinates (lower panels) and kinetic energies (middle panels) for three different collision geometries as sketched in the upper most panels. All cases start with initial kinetic energy of 0.06 Ry and correspond to a deposition of $\mathrm{Na}_{6}$ on $\mathrm{Ar}_{384}$.


Fig. 6. Time evolution of deposition for $\mathrm{Na}_{8}$ (left part) and $\mathrm{Na}_{9}^{+}$on $\mathrm{Ar}_{384}$ surface at impact energy of $0.001 \mathrm{Ry}$ per $\mathrm{Na}$ ion. Shown are the $z$ coordinates (lower) as well as the kinetic energies of $\mathrm{Na}$ ions and $\mathrm{Ar}$ atoms (upper). 
cluster in full detail by time-dependent density-functional theory, the $\mathrm{Na}^{+}$ions by classical dynamics, and the $\mathrm{Ar}$ atoms also at a classical level with position and dipole polarization as dynamical variables. We have studied in detail the influence of initial conditions on the deposition process, varying substrate activity, impact energy, collisional geometry, and size as well as charge of the Na cluster.

We have found that the gross features of deposition dynamics are much the same in all cases. The Ar system acts always as a very efficient and smooth stopper which absorbs almost all (more than 90\%) of the impact energy and thus yields capture over a broad range of energies. Cluster reflection, which is typical for hard surfaces, can be forced only at such high impact energies that the surface is destroyed. The impinging $\mathrm{Na}$ cluster is stopped at first impact and its energy is transferred to the Ar system in less than $0.5 \mathrm{ps}$. The transfer is almost complete leaving less than $10 \%$ of the energy to the cluster. The transferred energy is distributed also very quickly over all Ar atoms, propagating like a sound wave with speed of sound through the medium. The full thermalization over all ionic and atomic degrees of freedom proceeds on a slow scale with time of 5 ps and more (increasing with increasing impact energy). The collisional geometry (initial orientation and horizontal position relative to the surface) has no effect on the overall dynamical pattern, but determines subtle details of the captured state as, e.g., remaining cluster energy or distance to the surface. Cluster size and shape seem to make little difference. Cluster charge however can change a lot. It enhances overall excitation as well as binding of the cluster to the surface and produces a much tighter final configuration.

Acknowledgments: This work was supported by the DFG, project nr. RE 322/10-1, the French-German exchange program PROCOPE nr. 07523TE, the CNRS Programme "Matériaux" (CPR-ISMIR), Institut Universitaire de France, the Humboldt foundation and a Gay-Lussac price.

\section{A The Na-Ar energy functional in detail}

The degrees of freedom of the model are the wavefunctions of valence electrons of the metal cluster, $\left\{\varphi_{n}(\mathbf{r}), n=\right.$ $\left.1 \ldots N_{\mathrm{el}}\right\}$, the coordinates of the cluster's $\mathrm{Na}^{+}$ion cores, $\left\{\mathbf{R}_{I}, I=1 \ldots N_{\text {ion }}\right\}$, of the Ar atoms cores $\left.\operatorname{Ar}^{Q+}\right),\left\{\mathbf{R}_{a}, a=\right.$ $\left.1 \ldots N_{\mathrm{Ar}}\right\}$, and of the Ar valence clouds, $\left\{\mathbf{R}_{a}^{\prime}, a=1 \ldots N_{\mathrm{Ar}}\right\}$. ¿From the given total energy, the corresponding equations of motion are derived in a standard manner by variation. This leads to the (time-dependent) Kohn-Sham equations for the one single-particle wavefunctions $\varphi_{n}(\mathbf{r})$ of the cluster electrons, and Hamiltonian equations of motion for the other three degrees of freedom, thus treated by classical molecular dynamics (MD). For the valence cluster electrons, we use a density functional theory at the level of the time-dependent local-density approximation (TDLDA), augmented with an average-density self-interaction correction
(ADSIC) 45. The density of these electrons is given naturally as defined in mean-field theories and reads $\rho_{\mathrm{el}}(\mathbf{r})=$ $\sum_{n}\left|\varphi_{n}(\mathbf{r})\right|^{2}$. An Ar atom is described by two constituents with opposite charge, positive Ar core and negative Ar valence cloud, which allows a correct description of polarization dynamics. In order to avoid singularities, we associate a smooth (Gaussian) charge distribution to both constituents having width $\sigma_{\mathrm{Ar}}$ of the order of the p shell "size" in Ar atoms, in the spirit of [33]:

$$
\begin{aligned}
& \rho_{\mathrm{Ar}, a}(\mathbf{r})=\frac{e Q}{\pi^{3 / 2} \sigma_{\mathrm{Ar}}^{3}} \times \\
& \quad \times\left[\exp \left(-\frac{\left(\mathbf{r}-\mathbf{R}_{a}\right)^{2}}{\sigma_{\mathrm{Ar}}^{2}}\right)-\exp \left(-\frac{\left(\mathbf{r}-\mathbf{R}_{a}^{\prime}\right)^{2}}{\sigma_{\mathrm{Ar}}^{2}}\right)\right] .
\end{aligned}
$$

The corresponding Coulomb potential exerted by the Ar atoms is related to the charge distribution (11) by the Poisson equation, and reads:

$$
\begin{aligned}
V_{\mathrm{Ar}, a}^{(\mathrm{pol})}(\mathbf{r})=e^{2} Q\left[\frac{\operatorname{erf}\left(\left|\mathbf{r}-\mathbf{R}_{a}\right| / \sigma_{\mathrm{Ar}}\right)}{\left|\mathbf{r}-\mathbf{R}_{a}\right|}\right. & \left.\begin{array}{r}
\operatorname{erf}\left(\left|\mathbf{r}-\mathbf{R}_{a}^{\prime}\right| / \sigma_{\mathrm{Ar}}\right) \\
\left|\mathbf{r}-\mathbf{R}_{a}^{\prime}\right|
\end{array}\right],
\end{aligned}
$$

where $\operatorname{erf}(r)=\frac{2}{\sqrt{\pi}} \int_{0}^{r} \mathrm{~d} x e^{-x^{2}}$ stands for the error function. As for the $\mathrm{Na}^{+}$ions, their dynamical polarizability is neglected and we treat them simply as charged point particles.

The total energy of the system is composed as:

$$
E_{\text {total }}=E_{\text {Nacluster }}+E_{\mathrm{Ar}}+E_{\text {coupl }}+E_{\mathrm{VdW}} .
$$

The energy of the Na cluster $E_{\text {Nacluster }}$ consists out of TDLDA (with SIC) for the electrons, MD for ions, and a coupling of both by soft, local pseudo-potentials, for details see $31,30,29$. The Ar system and its coupling to the cluster are described by

$$
\begin{gathered}
E_{\mathrm{Ar}}=\sum_{a} \frac{\mathbf{P}_{a}^{2}}{2 M_{\mathrm{Ar}}}+\sum_{a} \frac{\mathbf{P}_{a}^{\prime 2}}{2 m_{\mathrm{Ar}}}+\frac{1}{2} k_{\mathrm{Ar}}\left(\mathbf{R}_{a}^{\prime}-\mathbf{R}_{a}\right)^{2} \\
+\sum_{a<a^{\prime}}\left[\int \mathrm{d} \mathbf{r} \rho_{\mathrm{Ar}, a}(\mathbf{r}) V_{\mathrm{Ar}, a^{\prime}}^{(\mathrm{pol})}(\mathbf{r})+V_{\mathrm{Ar}, \mathrm{Ar}}^{(\mathrm{core})}\left(\mathbf{R}_{a}-\mathbf{R}_{a^{\prime}}\right)\right] \\
E_{\mathrm{coupl}}=\sum_{I, a}\left[V_{\mathrm{Ar}, a}^{(\mathrm{pol})}\left(\mathbf{R}_{I}\right)+V_{\mathrm{Na}, \mathrm{Ar}}^{\prime}\left(\mathbf{R}_{I}-\mathbf{R}_{a}\right)\right] \\
+\int \mathrm{d} \mathbf{r} \rho_{\mathrm{el}}(\mathbf{r}) \sum_{a}\left[V_{\mathrm{Ar}, a}^{(\mathrm{pol})}(\mathbf{r})+W_{\mathrm{el}, \mathrm{Ar}}\left(\left|\mathbf{r}-\mathbf{R}_{a}\right|\right)\right] \\
E_{\mathrm{VdW}}=\frac{e^{2}}{2} \sum_{a} \alpha_{a}\left[\frac{\left(\int \mathrm{d} \mathbf{r} \mathbf{f}_{a}(\mathbf{r}) \rho_{\mathrm{el}}(\mathbf{r})\right)^{2}}{N_{\mathrm{el}}}\right. \\
\mathbf{f}_{a}(\mathbf{r})=\nabla \frac{\left.\mathrm{d} \mathbf{r} \mathbf{f}_{a}(\mathbf{r})^{2} \rho_{\mathrm{el}}(\mathbf{r})\right]}{\left|\mathbf{r}-\mathbf{R}_{a}\right|}
\end{gathered}
$$

The Van der Waals interaction between cluster electrons and Ar dipoles is written in Eq. (6) as a correlation 
from the dipole excitation in the Ar atom coupled with a dipole excitation in the cluster. We exploit that the plasmon frequency $\omega_{\text {Mie }}$ is far below the excitations in the Ar atom. This simplifies the term to the variance of the dipole operator in the cluster, using the regularized dipole operator $\mathbf{f}_{a}$, defined in Eq.(17), corresponding to the smoothened Ar charge distributions 22,46 . The full dipole variance is simplified in terms of the local variance.

The interaction of one Ar atom with the other constituents (Ar atoms, $\mathrm{Na}^{+}$ions, cluster electrons) results from the balance between a strong repulsive core potential that falls off exponentially and an equally strong attraction from dipole polarizability. The (most important) polarization potential, $V_{\mathrm{Ar}, a}^{(\mathrm{pol})}$, is described by a valence electron cloud oscillating against the Ar core ion. Its parameters are the effective charge of valence cloud $Q$, the effective mass of valence cloud $m_{\mathrm{Ar}}=Q m_{\mathrm{el}}$, the restoring force for dipoles $k_{\mathrm{Ar}}$, and the width of the core and valence clouds $\sigma_{\mathrm{Ar}}$. The $Q$ and $k_{\mathrm{Ar}}$ are adjusted to reproduce experimental data on dynamical polarizability $\alpha_{D}(\omega)$ of the Ar atom at low frequencies, namely the static limit $\alpha_{D}(\omega=0)$ and the second derivative of $\alpha_{D}^{\prime \prime}\left(\omega^{\prime \prime}=0\right)$. The width $\sigma_{\mathrm{Ar}}$ is determined consistently such that the restoring force from the folded Coulomb force (for small displacements) reproduces the spring constant $k_{\mathrm{Ar}}$.

The short range repulsion is provided by the various core potentials. For the Ar-Ar core interaction in Eq. (4), we employ a Lennard-Jones type potential with parameters reproducing binding properties of bulk Ar :

$$
V_{\mathrm{Ar}, \mathrm{Ar}}^{(\mathrm{core}}(R)=e^{2} A_{\mathrm{Ar}}\left[\left(R_{\mathrm{Ar}} / R\right)^{12}-\left(R_{\mathrm{Ar}} / R\right)^{6}\right] \text {. }
$$

The Na-Ar core potential $V_{\mathrm{Na}, \mathrm{Ar}}^{\prime}$ in Eq. (5) is chosen according to [34, within properly avoiding double counting of the dipole polarization-potential, hence the following form :

$$
\begin{aligned}
V_{\mathrm{Na}, \mathrm{Ar}}^{\prime} & (R)=e^{2}\left[A_{\mathrm{Na}} \frac{e^{-\beta_{\mathrm{Na}} R}}{R}\right. \\
& \left.-\frac{2}{1+e^{\alpha_{\mathrm{Na}} / R}}\left(\frac{\alpha_{\mathrm{Ar}}}{2 R^{4}}+\frac{C_{\mathrm{Na}, 6}}{R^{6}}+\frac{C_{\mathrm{Na}, 8}}{R^{8}}\right)\right] \\
+ & e^{2} \frac{\alpha_{\mathrm{Ar}}}{2 R^{3}} \mathbf{R} \cdot \nabla_{\mathbf{R}} \frac{\operatorname{erf}\left(R / \sqrt{2} \sigma_{\mathrm{Ar}}\right)}{R} .
\end{aligned}
$$

Finally the pseudo-potential $W_{\mathrm{el}, \mathrm{Ar}}$ in Eq. (5) for the electronAr core repulsion has been modeled according to the proposal of 33] :

$$
W_{\mathrm{el}, \mathrm{Ar}}(r)=e^{2} \frac{A_{\mathrm{el}}}{1+e^{\beta_{\mathrm{el}}\left(r-r_{\mathrm{el}}\right)}}
$$

The various contributions are calibrated from independent sources, with a final fine tuning to the NaAr dimer (bond length, binding energy, and optical excitation spectrum) modifying only the term $W_{\text {elAr }}$. The parameters are summarized in table 1. The third column of the table indicates the source for the parameters.

\section{References}

1. H. Brune, Metal Clusters at Surfaces, Structures (Springer, Berlin, 2000), p. 67

2. Euro. Phys. J. D 9 (1999), proceedings of ISSPIC 9, Lausanne 1998

3. Euro. Phys. J. D 16 (2001), proceedings of ISSPIC 10, Atlanta 2000

4. Euro. Phys. J. D 24 (2003), proceedings of ISSPIC 11, Strasbourg 2002

5. Euro. Phys. J. D 34 (2005), proceedings of ISSPIC 12, Nanjing 2004

6. C. Binns, Surf. Sci. Rep. 44, 1 (2001)

7. W. Harbich, Metal Clusters at Surfaces, Structures (Springer, Berlin, 2000), p. 107

8. Y.Z. Li, R. Reifenberger, R.P. Andres, Surf. Sci. 250, 1 (1991)

9. D.M. Schaefer, A. Patil, R.P. Andres, R. Reifenberger, Phys. Rev. B 51, 5322 (1995)

10. C. Kuhrt, M. Harsdorff, Surf. Sci. 245, 252 (1995)

11. R.N. Barnett, U. Landmann, Phys. Rev. Lett. 67, 727 (1991)

12. H.P. Cheng, U. Landmann, Science 260, 1304 (1991)

13. H. Häkkinen, R.N. Barnett, U. Landmann, Europhys. Lett. 28, 263 (1994)

14. H. Häkkinen, M. Manninen, Europhys. Lett. 34, 177 (1996)

15. C. Kohl, B. Montag, P.G. Reinhard, Z. f. Physik D 38, 81 (1996)

16. C. Kohl, P.G. Reinhard, Z. f. Physik D 39, 225 (1997)

17. C. Kohl, P.G. Reinhard, E. Suraud, Euro. Phys. J. D 11, $115(2000)$

18. A. Ipatov, E. Suraud, , P.G. Reinhard, Int. J. Mol. Sci. 4, 301 (2003)

19. A. Ipatov, P.G. Reinhard, , E. Suraud, Eur. Phys. J. D 30, $65(2004)$

20. B. Gervais, E. Giglio, E. Jaquet, A. Ipatov, P.G. Reinhard, E. Suraud, J.Chem.Phys. 121, 8466 (2004)

21. F. Fehrer, Ph.D. thesis, Universität Erlangen/Nürnberg (2006)

22. F. Fehrer, M. Mundt, P.G. Reinhard, E. Suraud, Ann. Phys. (Leipzig) 14, 411 (2005)

23. M.J. Field, P.A. Bash, M. Karplus, J. Comp. Chem. 11, $700(1990)$

24. J. Gao, Acc. Chem. Res. 29, 298 (1996)

25. N. Gresh, D.R. Garmer, J. Comp. Chem. 17, 1481 (1996)

26. P.J. Mitchell, D. Fincham, J. Phys.: Condens. Matter 5, 1031 (1993)

27. A. Nasluzov, K. Neyman, U. Birkenheuer, N. Rösch, J. Chem. Phys. 115, 17 (2001)

28. F. Fehrer, P.G. Reinhard, E. Suraud, E. Giglio, B. Gervais, A. Ipatov, Appl. Phys. A 82, 151 (2005)

29. P.G. Reinhard, E. Suraud, Introduction to Cluster Dynamics (Wiley, New York, 2003)

30. F. Calvayrac, P.G. Reinhard, E. Suraud, C.A. Ullrich, Phys. Rep. 337, 493 (2000)

31. S. Kümmel, M. Brack, P.G. Reinhard, Euro. Phys. J. D 9 , 149 (1999)

32. B.G. Dick, A.W. Overhauser, Phys. Rev. 112, 90 (1958)

33. F. Duplàe, F. Spiegelmann, J. Chem. Phys. 105, 1492 (1996)

34. G.R. Ahmadi, J. Almlöf, J. Roegen, Chem. Phys. 199, 33 (1995) 


\begin{tabular}{|l|l|l|}
\hline$V_{\mathrm{Ar}, a}^{(\mathrm{pol})}$ & $q_{\mathrm{Ar}}=\frac{\alpha_{\mathrm{Ar}} m_{\mathrm{el}} \omega_{0}^{2}}{e^{2}}, k_{\mathrm{Ar}}=\frac{e^{2} q_{\mathrm{Ar}}^{2}}{\alpha_{\mathrm{Ar}}}, m_{\mathrm{Ar}}=q_{\mathrm{Ar}} m_{\mathrm{el}}$ & $\begin{array}{l}\alpha_{\mathrm{Ar}}=11.08 \mathrm{a}_{0}^{3} \\
\omega_{0}=1.755 \mathrm{Ry}\end{array}$ \\
\hline$W_{\mathrm{elAr}}$ & $A_{\mathrm{el}}=0.47, \beta_{\mathrm{el}}=1.6941 / \mathrm{a}_{0}, r_{\mathrm{el}}=2.2 \mathrm{a}_{0}$ & fit to NaAr dimer [46],47] \\
\hline$V_{\mathrm{ArAr}}^{(\mathrm{core})}$ & $A_{\mathrm{Ar}}=1.367 * 10^{-3} \mathrm{Ry}, R_{\mathrm{Ar}}=6.501 \mathrm{a}_{0}$ & fit to bulk Ar \\
\hline$V_{\mathrm{ArNa}}^{\prime}$ & $\beta_{\mathrm{Na}}=1.7624 \mathrm{a}_{0}^{-1}, \alpha_{\mathrm{Na}}=1.815 \mathrm{a}_{0}, A_{\mathrm{Na}}=334.85$ & \\
& $C_{\mathrm{Na}, 6}=52.5 \mathrm{a}_{0}^{6}, C_{\mathrm{Na}, 8}=1383 \mathrm{a}_{0}^{8}$ & after [34] \\
\hline
\end{tabular}

Table 1. Parameters for the various model potentials.

35. B. Montag, P.G. Reinhard, Phys. Lett. A 193, 380 (1994)

36. B. Montag, P.G. Reinhard, Z. f. Physik D 33, 265 (1995)

37. W. Harbich, S. Fedrigo, F. Meyer, D. Lindsay, J. Lignires, J.C. Rivoal, D. Kreisle, J. Chem. Phys. 93, 8535 (1990)

38. T. Irawan, D. Boecker, F. Ghaleh, B. v Issendorf, H. Hövel, Appl. Phys. A 82, 81 (2005)

39. P.M. Dinh, F. Fehrer, G. Bousquet, P.G. Reinhard, E. Suraud (2007), preprint

40. W. Wahl, Proc. Roy. Soc. (London) A 87, 371 (1912)

41. G.L. Pollack, Rev. Mod. Phys. 36, 748 (1964)

42. W.A. de Heer, Rev. Mod. Phys. 65, 611 (1993)

43. M. Brack, Rev. Mod. Phys. 65, 677 (1993)

44. C. Sieber, W. Harbich, K.H. Meiwes-Broer, C. Félix, Chem. Phys. Lett. 433, 32 (2006)

45. C. Legrand, E. Suraud, P.G. Reinhard, J. Phys. B 35, 1115 (2002)

46. M. Gross, F. Spiegelmann, J. Chem. Phys. 108, 4148 (1998)

47. M.B. El Hadj Rhouma, H. Berriche, Z.B. Lakhdar, F. Spiegelman, J. Chem. Phys. 116, 1839 (2002) 